6. European Association for the Study of the Liver. EASL Clinical Practice Guidelines on nutrition in chronic liver disease. J Hepatol 2019;70(1):172-193.

7. Plauth $M$, Bernal W, Dasarathy $S$, et al. ESPEN guideline on clinical nutrition in liver disease. Clin Nutr 2019;38(2):485-521.

8. Arora S, Mattina C, Catherine M, et al. PMO-040 the development and validation of a nutritional prioritising tool for use in patients with chronic liver disease. Gut 2012;61:A90

\section{P048 GEOGRAPHIC VARIABILITY IN RATES OF INTENSIVE CARE UNIT ADMISSION IN PATIENTS WITH CHRONIC LIVER DISEASE AND CRITICAL COVID-19: INTERNATIONAL REGISTRY DATA}

${ }^{1}$ Thomas Marjot* ${ }^{2}$ Andrew Moon, ${ }^{3}$ Matthew Armstrong, ${ }^{4}$ Ignacio García-Juárez, ${ }^{5}$ Anand Kulkarni, ${ }^{6}$ Steven Masson, ${ }^{7}$ Nneka Ufere, ${ }^{8}$ David Wong, COVID-Hep \& SECURELiver contributors ${ }^{1,9}$, ${ }^{2}$ Luke Baldelli, ${ }^{2}$ Alfred Barritt, ${ }^{1}$ Eleanor Barnes, ${ }^{9} \mathrm{G}$ wilym Webb. ${ }^{1}$ Oxford Liver Unit, Translational Gastroenterology Unit, Oxford University Hospitals NHS Foundation Trust, University of Oxford, Oxford, UK; ${ }^{2}$ Division of Gastroenterology and Hepatology, University of North Carolina, Chapel Hill, North Carolina, USA; ${ }^{3}$ Liver Unit, Queen Elizabeth Hospital Birmingham, Birmingham, UK; ${ }^{4}$ Department of Gastroenterology, Instituto Nacional de Ciencias Médicas y Nutrición Salvador Zubirán, Mexico City, Mexico; ${ }^{5}$ Dept. of Hepatology and Liver Transplantation, Asian Institute of Gastroenterology Hospitals, Gachibowli, Hyderabad, India; 'Liver Transplant Unit, Freeman Hospital, The Newcastle upon Tyne Hospitals NHS Foundation Trust, Newcastle upon Tyne, UK; ' Liver Center, Gastrointestinal Division, Massachusetts General Hospital, Harvard Medical School, Boston, Massachusetts, USA; ${ }^{8}$ Toronto Centre for Liver Disease, University Health Network, University of Toronto, Canada; ${ }^{9}$ Cambridge Liver Unit, Addenbrooke's Hospital, Cambridge University Hospitals, Cambridge, UK

10.1136/gutjnl-2021-BASL.57

Background and Aims The COVID-19 pandemic has provided a unique opportunity to evaluate global intensive care unit (ICU) admission practices for a common indication. Patients with chronic liver disease (CLD) and cirrhosis may also have limited or variable access to ICU. We aimed to describe international ICU admission rates and outcomes in patients with CLD and critical COVID-19.

Methods Data were combined from two international registries (SECURE-Liver and COVID-Hep) for patients with CLD and COVID-19 which was deemed severe enough to require ICU by the reporting clinician. Rates of ICU admission or decline, and respective outcomes were compared by country.

Results Between 25th March 2020 and 3rd February 2021, 319 patients with CLD and COVID-19 from 27 countries were judged to require ICU. The proportion of patients ultimately accepted to ICU varied according to country (figure
1A), although mortality following ICU admission was similar by country (figure 1B). Factors associated with being declined ICU admission included advancing age, cirrhosis, alcohol related liver disease, and UK origin. To explore national differences further, we compared cases from the USA and UK, the two greatest contributing countries. Rates of ICU admission differed significantly between the USA and UK [77/79 $(95 \%)$ vs. $22 / 77$ (29\%); p<0.001]. However, there were no differences in mortality in those admitted to ICU $(42 / 75$ [56\%] vs. $10 / 22$ [45\%]; $\mathrm{p}=0.468$; figure $1 \mathrm{~B})$, or after receiving invasive ventilation $(29 / 59$ [49\%] vs. 9/17 [53\%]; $\mathrm{p}=1.000)$. There were also no differences in age, sex, Charlson Comorbidity Index, or baseline liver disease severity between countries, both in those requiring and admitted to ICU. This included comparable rates of cirrhosis (53/79 [67\%] vs. $56 / 77[72 \%] ; p=0.723)$. Only four USA patients were declined ICU admission of whom $2(50 \%)$ died, whereas 55 UK patients were declined ICU admission of whom 51 (93\%) died. In both USA and UK cohorts, the reason for not admitting patients to ICU was due to this being deemed inappropriate by the responsible clinician, except for one case in both countries where no ICU bed was available. Notably, information relating to patient wishes, long-term outcomes in survivors, and granular detail regarding organ support requirements were not available.

Conclusion Patients with CLD and critical COVID-19 were over 3-times more likely to be admitted to ICU in the USA than the UK despite having similar baseline characteristics. However, the rates of mortality following ICU admission were comparable between the two countries. The differing thresholds for escalation to ICU but similar post admission outcomes warrants further discussion.

\section{P049 DECOMPENSATED LIVER DISEASE: A QUALITY IMPROVEMENT PROJECT}

Rebecca Osborne*, Geeta Prasad. Wythenshawe Hospital, Manchester, UK

\subsection{6/gutjnl-2021-BASL.58}

Decompensated liver disease is considered a medical emergency with an associated high morbidity and mortality (10$20 \%)^{1}$. It is a common presentation to hospital with multi-

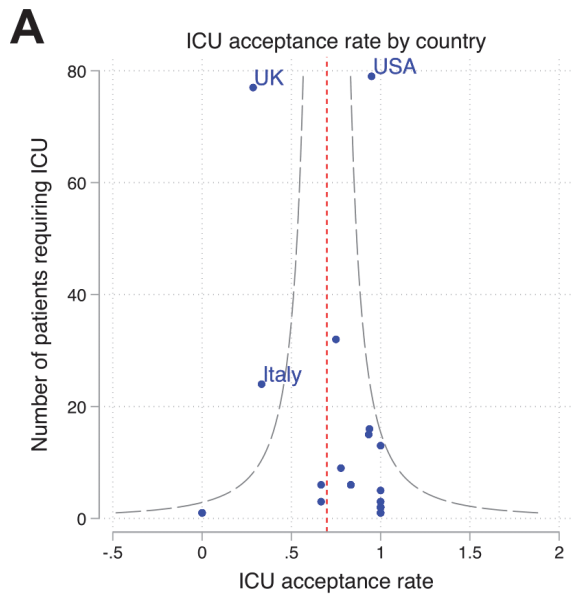

B

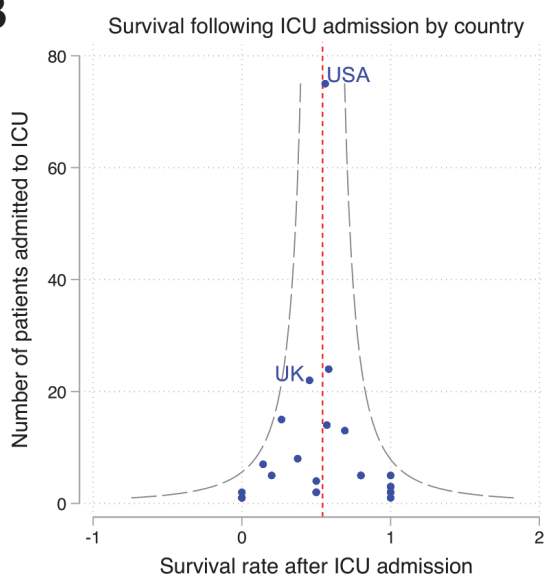

